\title{
Integrative analysis of the gastric cancer long non-coding RNA-associated competing endogenous RNA network
}

\author{
YUYOU JIANG $^{1}$, XIANQIN ZHANG ${ }^{2}$, LI RONG $^{3}$, YI HOU $^{4}$, JING SONG $^{1}$, \\ WANFENG ZHANG ${ }^{5}$, MIN HE ${ }^{1}$, YAN XIE ${ }^{1}$, YUE LI ${ }^{1}$ and FANGZHOU SONG ${ }^{1}$
}

\begin{abstract}
${ }^{1}$ Basic Medical College, Chongqing Medical University, Chongqing 400016; ${ }^{2}$ School of Basic Medical Sciences, Chengdu Medical College, Chengdu, Sichuan 610500; ${ }^{3}$ Department of Infectious Disease, Chongqing Public Health Medical Center, Chongqing 400036; ${ }^{4}$ Experimental Teaching and Management Center, Chongqing Medical University, Chongqing 401331; ${ }^{5}$ Department of Bioinformatics, Chongqing Medical University, Chongqing 400016, P.R. China
\end{abstract}

Received November 25, 2020; Accepted March 10, 2021

DOI: $10.3892 / \mathrm{ol} .2021 .12717$

\begin{abstract}
Gastric cancer (GC) is a common type of cancer, and identification of novel diagnostic biomarkers associated with this disease is important. The present study aimed to identify novel diagnostic biomarkers associated with the prognosis of GC, using an integrated bioinformatics approach. Differentially expressed long non-coding RNAs (lncRNAs) associated with GC were identified using Gene Expression Omnibus datasets (GSE58828, GSE72305 and GSE99416) and The Cancer Genome Atlas database. A competing endogenous RNA network that incorporated five lncRNAs [long intergenic non-protein coding RNA 501 (LINC00501), LINC00365, SOX21 antisense divergent transcript 1 (SOX21-AS1), GK intronic transcript 1 (GK-IT1) and DLEU7 antisense RNA 1 (DLEU7-AS1)], 29 microRNAs and 114 mRNAs was constructed. Gene Ontology and protein-protein interaction network analyses revealed that these IncRNAs may be involved in 'biological regulation', 'metabolic process', 'cell communication', 'developmental process', 'cell proliferation', 'reproduction' and the 'cell cycle'. The results of receiver operating characteristic curve analysis demonstrated that LINC00501 (AUC=0.819), LINC00365 (AUC=0.580), SOX21-AS1 (AUC=0.736), GK-IT1 $(\mathrm{AUC}=0.823)$ and DLEU7-AS1 (AUC=0.932) had the potential to become valuable diagnostic biomarkers for GC. Associations with clinicopathological characteristics demonstrated that LINC00501 expression was significantly associated with sex $(\mathrm{P}=0.015)$ and tumor grade $(\mathrm{P}=0.022)$. Furthermore, LINC00365 expression was significantly associated with
\end{abstract}

Correspondence to: Professor Fangzhou Song, Basic Medical College, Chongqing Medical University, 1 Yixueyuan Road, Yuzhong, Chongqing 400016, P.R. China

E-mail: fzsongcq@163.com

Key words: gastric cancer, long non-coding RNA, The Cancer Genome Atlas, Gene Expression Omnibus, competing endogenous RNA lymph node metastasis $(\mathrm{P}=0.025)$. Gene set enrichment analysis revealed that LINC00501, LINC00365 and SOX21-AS1 were enriched in signaling pathways associated with GC. Reverse transcription-quantitative PCR analysis demonstrated that LINC00501 expression $(\mathrm{P}=0.043)$ was significantly upregulated in $\mathrm{GC}$ tissues, whereas the expression levels of LINC00365 $(\mathrm{P}=0.033)$ and SOX21-AS1 $(\mathrm{P}=0.037)$ were significantly downregulated in GC tissues. Taken together, the results of the present study suggest that LINC00501, LINC00365, SOX21-AS1, GK-IT1 and DLEU7-AS1 may be used as novel diagnostic biomarkers for GC, and may be functionally associated with GC development and progression.

\section{Introduction}

Among the most common types of cancer, gastric cancer (GC) was the second leading cause of cancer-associated mortality worldwide until 2018, with $\sim 1$ million new cases of GC and 782,685 GC-associated mortalities occurring in 2018 (1). Radical surgery remains the most reliable treatment for GC (2). Due to advancements in surgical techniques, chemotherapy and radiotherapy, the 5-year survival rate of patients with early stage GC is $>95 \%$ (2). However, early stage GC is often asymptomatic and rarely detected, and thus, $>70 \%$ of patients with GC eventually develop advanced GC and may be ineligible for surgical resection (2). Thus, it is important to identify novel biomarkers associated with GC to improve the diagnosis of this deadly disease.

Long non-coding RNAs (lncRNAs) are RNA molecules that are $>200$ nucleotides in length, lack protein coding potential, and can regulate the migration, survival and proliferation of cancer cells $(3,4)$. Several studies have reported that specific IncRNAs are dysregulated in GC, including urothelial cancer-associated 1 (5), antisense RNA in the INK4 locus (6) and AC093818.1 (7). At the functional level, lncRNAs serve as competing endogenous RNAs (ceRNAs) that bind to specific microRNAs (miRNAs/miRs) in a competitive manner, thereby upregulating the expression levels of miRNA target genes (8). Several studies have demonstrated the interactions between IncRNAs, miRNAs and mRNAs, which influence the development and progression of different types of cancer. For example, 
lncRNA HNF1A antisense RNA 1 binds to miR-30b-3p in $\mathrm{GC}$, which promotes activation of the PI3K/AKT signaling pathway (9). In addition, lncRNA DCST1 antisense RNA 1 regulates the survival, proliferation and invasion of GC cells by competitively binding and sequestering miR-605-3p (10). It has been reported that lncRNA metallothionein $1 \mathrm{~J}$ pseudogene can control F-box and WD repeat domain containing 7 expression by competitively targeting miR-92a-3p in GC cells (11). Thus, broader analyses of 1ncRNA-miRNA-mRNA ceRNA networks may enable researchers to further understand complex GC-associated gene interactions. In addition, a ceRNA model can offer unique insights to understand the putative roles of several uncharacterized lncRNAs in GC development and progression.

In the present study, an integrated systematic analysis of lncRNAs and mRNA expression patterns in datasets of patients with GC was performed. Multiple publicly available Gene Expression Omnibus (GEO) datasets (GSE58828, GSE72305 and GSE99416) were normalized and integrated to identify genes that were differentially expressed between tumor tissues and adjacent normal tissues from patients with GC. In addition, The Cancer Genome Atlas (TCGA) database was used to confirm the differential expression profiles of lncRNAs in GC. A ceRNA network was constructed using these data. Gene Ontology (GO) and protein-protein interaction (PPI) analyses of mRNAs in the GC-related ceRNA network were performed to further investigate the association between the lncRNAs and GC in the ceRNA network. Receiver operating characteristic (ROC) curve analysis was performed to determine the diagnostic value of the central lncRNAs. Subsequently, associations with clinicopathological characteristics were assessed, and gene set enrichment analysis (GSEA) of the central lncRNAs was performed.

The present study aimed to identify novel biomarkers for the diagnosis of GC and provide insight into the mechanistic basis of GC development and progression.

\section{Materials and methods}

Microarray datasets. Studies from the GEO database (https://www.ncbi.nlm.nih.gov/geo) were considered eligible according to the following criteria: i) Studies with GC tissue samples; ii) studies with information on technology and platform used for studies. Based on these criteria, the GSE58828, GSE72305 (12) and GSE99416 (13) GC datasets were downloaded from the GEO database (14). Details of each microarray study are presented in Table I. GC RNA expression profile data were obtained from TCGA database (http://tcga-data. nci.nih.gov) (15). An overview of the bioinformatics analysis performed to assess the GEO and TCGA datasets is presented in Fig. S1.

Differential expression analysis. All three datasets were initially integrated to increase the overall sample numbers (19 tumor samples and 11 normal samples), and introduction of unreliable results was avoided by batch normalizing the three datasets, using the sva package (16) (version 3.38.0; http://www. bioconductor.org/packages/release/bioc/html/sva.html) and limma package (17) (version 3.46.0; https://bioconductor. org/packages/release/bioc/html/limma.html) in R software (version 3.5.1; www.R-project.org). Subsequently, tumor and normal tissue samples were compared using the limma package in R software (17), and differential RNA expression was detected using the following criteria: $\mid \log$ fold-change $(\mathrm{FC}) \mid>1$ and adjusted $\mathrm{P}<0.05$. A volcano map was constructed using the pheatmap package (version 1.0.12; https://cran.r-project. org/web/packages/pheatmap/index.html) in R software.

ceRNA network construction. A ceRNA network was constructed to visualize lncRNA-miRNA-mRNA interactions, based on the ceRNA model wherein lncRNAs can bind and sequester miRNAs, thereby altering their ability to influence mRNA translation (8). The miRcode database (version 11; http://www.mircode.org) (18) was used to identify intersecting lncRNA target miRNAs, while miRDB (version 5.0; http://mirdb.org), TargetScan 7.2 (version 7.2; http://www.targetscan.org) (19) and miRTarBase (version 7.0; http://mirtarbase.cuhk.edu.cn/php/download. php) (20) were used to predict interactions between miRNAs and mRNAs.

Genes identified in at least two databases were considered miRNA targets. The target mRNAs were further intersected with dysregulated mRNAs in GEO GC samples. Subsequently, the ceRNA network was constructed by combining the lncRNA-miRNA interactomes and miRNA-mRNA interactomes, which was visualized using Cytoscape software (version 3.6.1; https://cytoscape.org) (21).

GO and PPI analysis. To assess the functional relevance of differentially expressed lncRNAs in the present study, $\mathrm{GO}$ analysis of the molecular functions, cellular components and biological processes, for which these genes were enriched, was performed using Webgestalt 2019 (http://www.webgestalt. org) (22). In addition, PPI network data of the mRNAs were collected from the Search Tool for the Retrieval of Interacting Genes (STRING) database (version 10.0; http://string-db. org) (23), and the PPI network was constructed using Cytoscape software.

ROC curve analysis. ROC curve analysis was performed to determine the diagnostic values of the differentially expressed lncRNAs in the ceRNA network, using the survival ROC package (version 1.66.0; https://bioconductor.org/packages/ROC) in R software. An area under the curve (AUC) value of $>0.50$ indicated evidence for diagnosis.

Correlations between IncRNA expression and clinicopathological characteristics. To further evaluate the clinical value of the central IncRNAs in GC, the associations between lncRNA expression and clinicopathological characteristics, including age, sex, grade, stage, $\mathrm{T}$ classification, $\mathrm{N}$ classification, $\mathrm{M}$ classification of TCGA data were analyzed using the $\chi^{2}$ test. Unpaired Student's t-test was used to compare differences between two groups. The tumor grading system was based on the Goseki histological grading of GC (24).

GSEA. For GSEA, cut-off values for each lncRNA were determined based on the median expression levels in the TCGA-STAD database (https://portal.gdc.cancer.gov/projects/TCGA-STAD). The 'c2.cp.kegg.v7.0.symbols.gmt' dataset 
Table I. Details of gastric cancer studies and associated microarray datasets from the Gene Expression Omnibus database.

\begin{tabular}{|c|c|c|c|c|c|c|c|c|c|}
\hline \multirow[b]{2}{*}{ Series accession no. } & \multirow[b]{2}{*}{ Platform } & \multicolumn{3}{|c|}{ Sample size, $\mathrm{n}$} & \multirow[b]{2}{*}{ DEGs, $\mathrm{n}$} & \multirow[b]{2}{*}{ Upregulated, $\mathrm{n}$} & \multirow[b]{2}{*}{ Downregulated, $\mathrm{n}$} & \multicolumn{2}{|c|}{ Sex, n } \\
\hline & & Total & Tumor & Adjacent & & & & Female & Male \\
\hline GSE58828 & GPL15314 & 6 & 3 & 3 & 9,121 & 4,869 & 4,252 & 2 & 4 \\
\hline GSE72305 & GPL15314 & 12 & 10 & 2 & 324 & 108 & 216 & NA & NA \\
\hline GSE99416 & GPL16956 & 12 & 6 & 6 & 2,651 & 1,261 & 1,390 & 4 & 8 \\
\hline
\end{tabular}

DEGs, differentially expressed genes.

was used for 1,000 gene set permutations per analysis for each lncRNA, using GSEA software (version 4.0.1; http://software. broadinstitute.org/gsea/downloads.jsp). The present study focused on pathways with nominal P-value $<0.05$ and selected the most significantly enriched signaling pathways based on their size $>50$. The results were generated using the ggplot2 package (version 3.3.3; https://CRAN.R-project. org/package $=$ ggplot2) in R software.

Tissue sample collection. A total of 15 GC tumor tissues and paired adjacent normal tissues $(5 \mathrm{~cm}$ away from the tumor margin) were collected from patients with GC who underwent radical primary tumor excision at The First Affiliated Hospital of Chongqing Medical University (Chongqing, China) between December 2016 and January 2019. The inclusion criteria were as follows: i) Patients had received an initial diagnosis of GC according to the results of gastroscopy and pathological biopsy; ii) all patients with GC were evaluated according to TNM stage (25) and iii) there were no significant differences in blood routine test indexes, liver function indexes and renal function indexes of all patients with GC. The exclusion criteria were as follows: i) patients with poor general condition and unable to tolerate related examinations; ii) concurrently diagnosed with other tumors and patients with secondary GC and iii) patients with GC who had received radiotherapy, chemotherapy or other treatments prior to surgery. The patients included 9 men and 6 women, with a mean age of $64.6 \pm 4.9$ years (age range, 50-75 years). Tissue samples were stored at $-80^{\circ} \mathrm{C}$ until subsequent experimentation. The present study was approved by the Research Ethics Committee of the First Affiliated Hospital of Chongqing Medical University (approval no. 2016140) and written informed consent was provided by all patients prior to the study start.

$R T$-qPCR. A total of 15 pairs of tumor tissues and adjacent normal tissues from patients with GC were used for RT-qPCR validation. Total RNA was extracted from tissues using TRIzol ${ }^{\circledR}$ reagent (Tiangen Biotech Co., Ltd.). The concentration of RNA was measured using a Nanodrop 2000 instrument (Bio-Rad Laboratories, Inc.). Total RNA (500 ng RNA) was reverse transcribed into cDNA using the PrimeScript RT Reagent kit with gDNA Eraser (Takara Biotechnology Co., Ltd.). qPCR was subsequently performed using specific primers (TsingKe Biological Technology) and TB Green ${ }^{\circledR}$ Premix ExTaq ${ }^{\mathrm{TM}}$ II (Takara Biotechnology Co., Ltd.). The following primer sequences were used for
qPCR: LINC00501 forward, 5'-GAACAATGACCGGGG AACAG-3' and reverse, 5'-TTCTTCCTTTGTGCTTCC GC-3'; LINC00365 forward, 5'-AGCTGCTCATCCTTCCTC AG-3' and reverse, 5'-ACACAGGTGCCAAAATCCAC-3'; SOX21-AS1 forward, 5'-GAGGTGCTGCAGGAGAGT TA-3' and reverse, 5'-ACTCTCCACTCGCCTAAACC-3'; DLEU7-AS1 forward, 5'-AACAAATTTGGGGCACTG CT-3' and reverse, 5'-CACCAAAGCACGGAAGGTAG-3'; and GK-IT1 forward, 5'-CTGAGGTTGGGAGTTCGA GAC-3' and reverse, 5'-GGATTACAGGCATGAGCCAC-3'; and GAPDH forward, 5'-GGTCTCCTCTGACTTCAACA-3' and reverse, 5'-GTGAGGGTCTCTCTCTTCCT-3'. The temperature protocol for RT was as follows: $37^{\circ} \mathrm{C}$ for $15 \mathrm{~min}$, $85^{\circ} \mathrm{C}$ for $5 \mathrm{sec}$ and $4^{\circ} \mathrm{C}$ storage. The resulting cDNA product was stored at $-20^{\circ} \mathrm{C}$. The following thermocycling conditions were used for qPCR: Initial denaturation at $95^{\circ} \mathrm{C}$ for $3 \mathrm{~min}$, followed by 40 cycles of $95^{\circ} \mathrm{C}$ for $1 \mathrm{sec}$ and $58^{\circ} \mathrm{C}$ for $30 \mathrm{sec}$. IncRNA expression levels were calculated using the $2^{-\Delta \Delta C q}$ method (26) and normalized to the internal reference gene GAPDH

Statistical analysis. Statistical analysis was performed using R software (version 3.5.1; www.R-project.org). All experiments were performed in triplicate and data are presented as the mean \pm standard deviation. Paired Student's t-test was used to compare differences between lncRNA expression levels in tumor tissues and adjacent normal tissues. $\mathrm{P}<0.05$ was considered to indicate a statistically significant difference.

\section{Results}

Pooled analysis of GC-related gene expression profiles in the GEO datasets. Following reannotation, 25,476, 22,053 and 32,386 genes were obtained in the GSE58828 (GPL15314), GSE72305 (GPL15314) and GSE99416 (GPL16956) datasets, respectively. Genes that were differentially expressed between GC tissues and normal tissues ( $\mid \log \mathrm{FCl}>1$ and false discovery rate $<0.05$ ) were identified using the limma package. In total, 9,121 genes were differentially expressed in the GSE58828 dataset (4,869 upregulated genes and 4,252 downregulated genes), while 324 genes were differentially expressed in the GSE72305 dataset (108 upregulated genes and 216 downregulated genes) and 2,651 genes were differentially expressed in the GSE99416 dataset (1,261 upregulated genes and 1,390 downregulated genes). These differentially expressed 
GSE58828

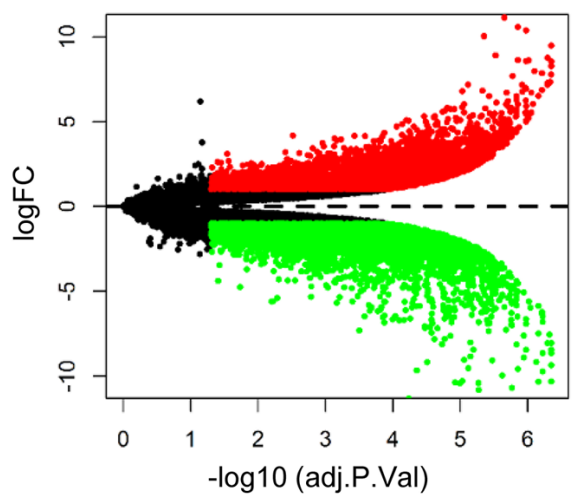

GSE72305

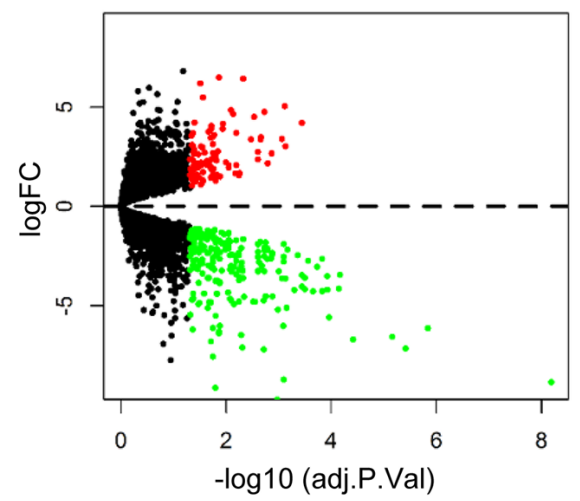

GSE99416

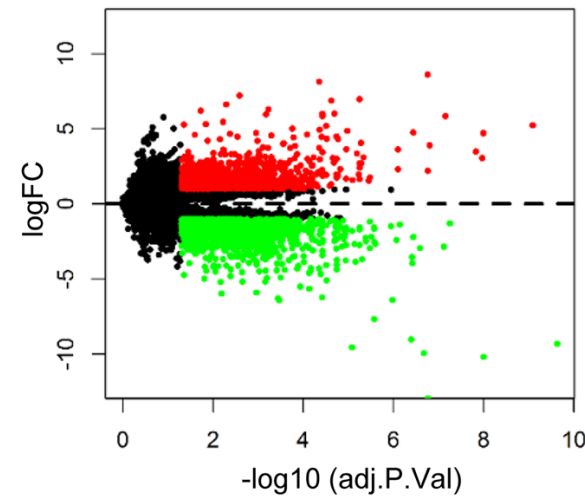

Figure 1. Differentially expressed gastric cancer-related genes in the Gene Expression Omnibus database. Red dots represent upregulated genes (adjusted $\mathrm{P}<0.05$ and $\log \mathrm{FC}>1$ ), green dots represent downregulated genes (adjusted $\mathrm{P}<0.05$ and $\log \mathrm{FC}<-1$ ); and black dots represent genes that were not identified to be significantly differentially expressed. FC, fold-change; adj.P.Val, adjusted P-value.
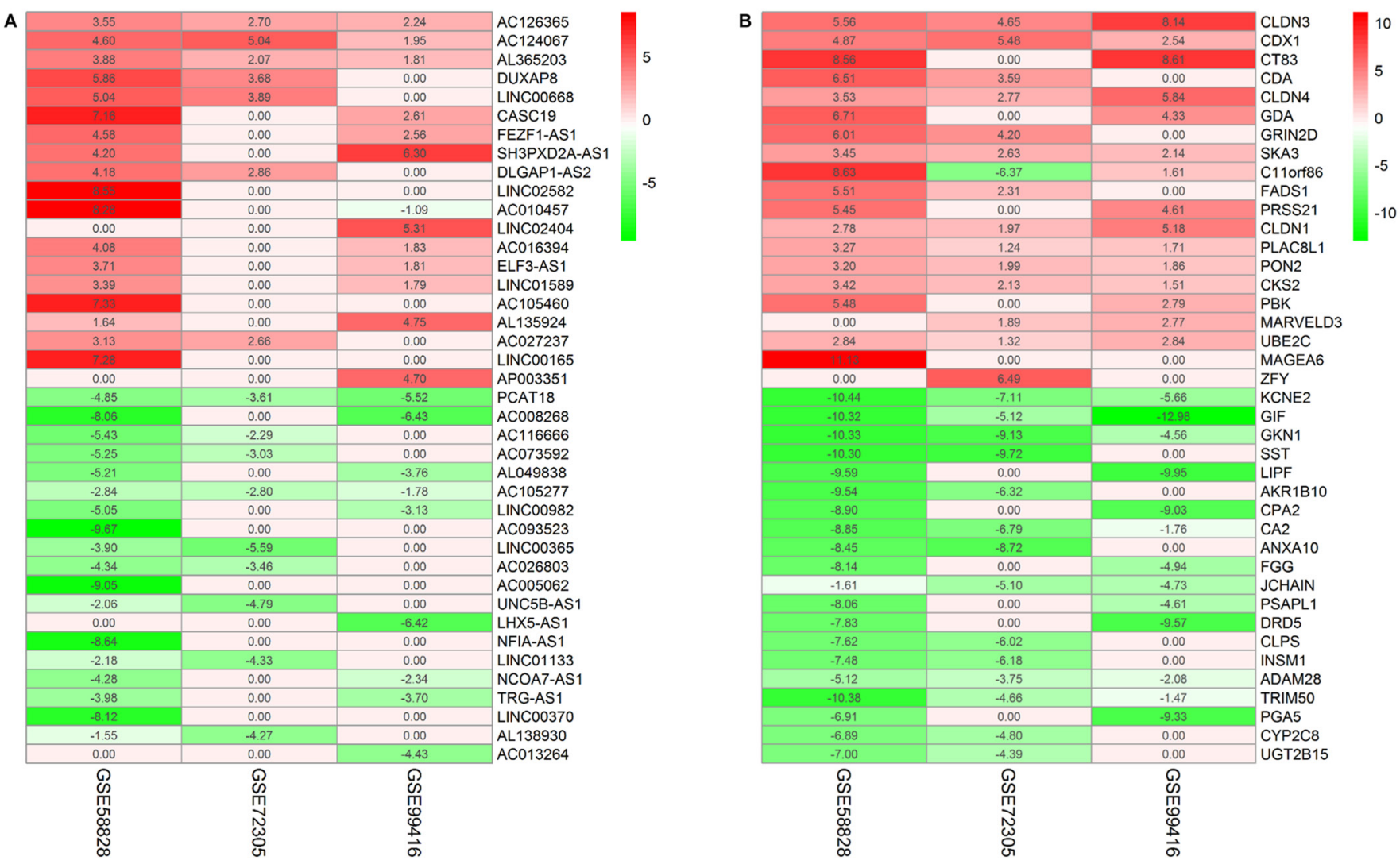

Figure 2. Top 20 gastric cancer-related differentially expressed genes in the Gene Expression Omnibus database. (A) Differentially expressed long non-coding RNAs. (B) Differentially expressed mRNAs. Red represents upregulated genes, while green represents downregulated genes. The number indicates the fold-change in each dataset.

genes are presented in volcano plots in Fig. 1. Following batch normalization, a total of 465 genes were differentially expressed between GC tissues and normal tissues. Overall, the present study identified 48 lncRNAs and 175 mRNAs that were upregulated, and 45 lncRNAs and 197 mRNAs that were downregulated in tumor tissues. The top 20 upregulated and downregulated lncRNAs (Fig. 2A) and mRNAs (Fig. 2B) in these datasets were identified, and further information regarding all the IncRNAs and mRNAs are presented in Tables SI and SII.
TCGA-based validation of differential IncRNA expression. GC sample RNA expression profiles were downloaded from TCGA database to validate the differential lncRNA expression profiles detected in the GEO dataset analysis. Comparisons of the GEO and TCGA datasets identified 25 differentially expressed lncRNAs (Table II).

ceRNA network. Based on the lncRNA-miRNA, circRNA-miRNA and miRNA-mRNA predicted interactions, a ceRNA network was constructed. The network incorporated 
Table II. Differentially expressed long non-coding RNAs in the Gene Expression Omnibus and The Cancer Genome Atlas databases.

\begin{tabular}{llrc}
\hline lncRNA & Regulation & logFC & Adjusted P-value \\
\hline PGM5-AS1 & Down & -3.601 & $<0.001$ \\
LHX5-AS1 & Down & -4.367 & $<0.001$ \\
LINC02163 & Up & 5.976 & $<0.001$ \\
DUXAP8 & Up & 3.582 & $<0.001$ \\
LINC00982 & Down & -2.251 & $<0.001$ \\
PCAT18 & Down & -3.098 & $<0.001$ \\
DLEU7-AS1 & Up & 2.599 & $<0.001$ \\
LINC00582 & Down & -2.196 & $<0.001$ \\
FEZF1-AS1 & Up & 4.654 & $<0.001$ \\
DLGAP1-AS2 & Up & 2.174 & $<0.001$ \\
LINC00365 & Down & -2.210 & $<0.001$ \\
NCOA7-AS1 & Down & -2.018 & $<0.001$ \\
LINC02404 & Down & -2.802 & $<0.001$ \\
LINC02447 & Down & -1.205 & $<0.001$ \\
SOX21-AS1 & Down & -2.296 & $<0.001$ \\
CASC19 & Up & 2.354 & $<0.001$ \\
UNC5B-AS1 & Down & -1.660 & $<0.001$ \\
LINC00501 & Up & 2.184 & $<0.001$ \\
LINC01133 & Down & -1.296 & $<0.001$ \\
LINC00853 & Up & 1.141 & $<0.001$ \\
LINC01985 & Down & -1.304 & $<0.001$ \\
GK-IT1 & Up & 1.337 & $<0.001$ \\
HRAT92 & Up & 1.366 & $<0.001$ \\
LINC01589 & Down & -1.061 & $<0.001$ \\
PICSAR & Up & 1.947 & $<0.001$ \\
\hline
\end{tabular}

lncRNA, long non-coding RNA; FC, fold-change.

five IncRNAs (LINC00501, LINC00365, SOX21-AS1, GK-IT1 and DLEU7-AS1), 29 miRNAs and 114 mRNAs (Fig. 3). Overall, 29 specific miRNAs were predicted to target five specific lncRNAs (Table III), and 26 specific miRNAs (hsa-miR-125b-5p, hsa-miR-129-5p and hsa-miR-455-5p did not target mRNAs from the intersecting specific mRNAs) were identified to interact with the 114 intersecting mRNAs (Table IV).

Functional enrichment and interaction analyses. To further investigate the association between the five IncRNAs and GC, GO and PPI network analyses of the 114 mRNAs within the constructed ceRNA network were performed. The results demonstrated that the mRNAs were involved in biological processes, including 'biological regulation', 'metabolic process', 'response to stimulus', 'multicellular organismal process', 'cell communication', 'developmental process', 'localization', 'cellular component organization', 'cell proliferation', 'reproduction' and 'multi-organism process growth' (Fig. 4A). The mRNAs were primarily enriched for cellular component terms, including 'membrane', 'cytosol', 'nucleus', 'endomembrane system', 'vesicle', 'extracellular space', 'membrane-enclosed lumen' and 'protein-containing complex' (Fig. 4B). For molecular functions terms, the mRNAs were concentrated in 'protein binding', 'ion binding', 'nucleotide binding', 'hydrolase activity', 'nucleic acid binding', 'transferase activity', 'enzyme regulator activity', 'molecular transducer activity', 'transporter activity' and 'lipid binding' (Fig. 4C).

A PPI network incorporating the mRNAs in the ceRNA network was constructed using the STRING database. The results demonstrated that the lncRNAs in the ceRNA network may be involved in regulating the cell cycle via cyclin B1 (CCNB1), family with sequence similarity 83 member D (FAM83D) and cell division cycle 6 (CDC6) in GC (Fig. 4D).

Diagnostic values of the lncRNAs. ROC curve analysis was performed to determine the diagnostic values of the central lncRNAs in the ceRNA network. The results generated AUC values of $0.819,0.932,0.823,0.580$ and 0.736 for LINC00501, DLEU7-AS1, GK-IT1, LINC00365 and SOX21-AS1, respectively (Fig. 5), which suggests that the five lncRNAs have diagnostic value for patients with GC.

Associations between lncRNAs and clinicopathological characteristics. The present study further assessed the clinical value of the central lncRNAs in GC, and associations between lncRNA expression and clinicopathological characteristics were analyzed using TCGA database. The results demonstrated that LINC00501 expression was significantly associated with sex $(\mathrm{P}=0.015$; Fig. 6A) and tumor grade $(\mathrm{P}=0.022$; Fig. 6B). Furthermore, LINC00365 expression was significantly associated with lymph node metastasis $(\mathrm{P}=0.025$; Fig. 6C). However, due to the limited sample size, data of associations between other lncRNAs expression and clinicopathological characteristics were non-significant (Table SIII).

Identification of IncRNA-associated signaling pathways. GSEA was performed to identify signaling pathways that are differentially activated in GC. The present study focused on signaling pathways that had nominal $\mathrm{P}<0.05$ and selected the most significantly enriched signaling pathways based on their SIZE $>50$. The results demonstrated that LINC00501 was enriched in the TGF-beta, mTOR, MAPK and WNT signaling pathways (Fig. 7A), while LINC00365 was enriched in the MAPK signaling pathway (Fig. 7B). In addition, SOX21-AS1 was enriched in the cell cycle (Fig. 7C). Taken together, these results suggest that these lncRNAs may modulate these signaling pathways, thereby regulate the development and progression of GC.

$R T-q P C R$ verification of differential lncRNA expression in $G C$ tissues. To confirm that the central lncRNAs were differentially expressed in GC tissues, RT-qPCR analysis was performed to detect their expression levels in 15 pairs of GC tissues and adjacent normal tissues. Consistent with the results of the GEO and TCGA dataset analyses, the results demonstrated that the expression levels of LINC00501, DLEU7-AS1 and GK-IT1 were upregulated in GC tumor tissues, whereas the expression levels of LINC00365 and SOX21-AS1 were downregulated in GC tumor tissues (Fig. 8). Notably, LINC00501 expression was increased 5.47-fold ( $\mathrm{P}=0.043)$, LINC00365 expression 


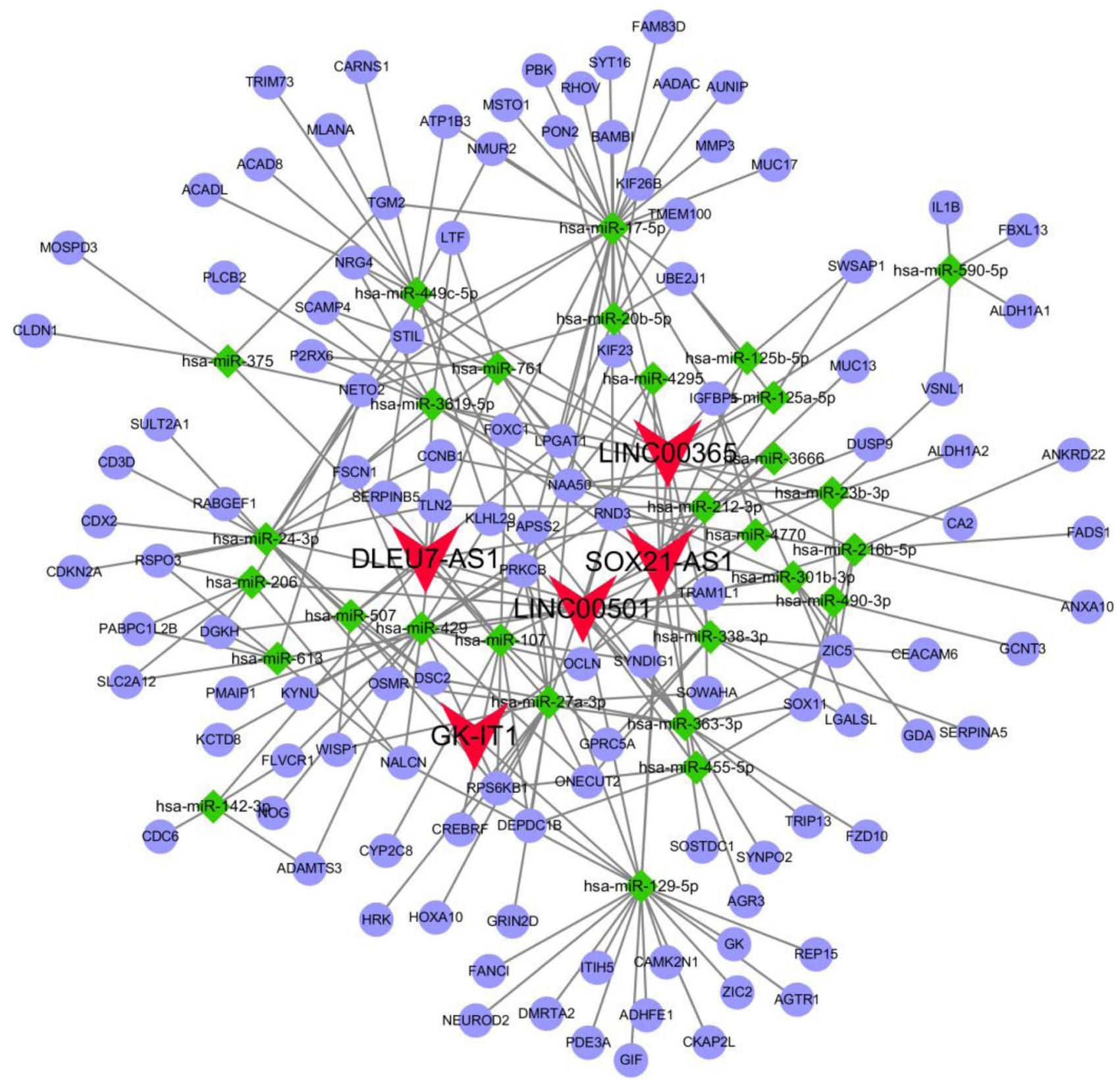

Figure 3. Competing endogenous RNA network. Red arrows represent long non-coding RNAs, green diamonds represent microRNAs and blue circles represent mRNAs. miR, microRNAs.

was decreased 3.67-fold $(\mathrm{P}=0.033)$ and SOX21-AS1 expression was decreased 3.24-fold $(\mathrm{P}=0.037)$. Although the differences in DLEU7-AS1 and GK-IT1 expression were not significant, they exhibited an upward trend consistent with the bioinformatics analyses.

\section{Discussion}

Increasing evidence suggest that lncRNAs are involved in the progression of different types of cancer (27-30). However, the etiology of GC remains partly unknown. In addition, the roles of lncRNAs in GC remain unclear. The present study analyzed multiple GEO and TCGA datasets to comprehensively identify lncRNAs associated with GC development or progression. By constructing a ceRNA network, performing functional enrichment analyses and ROC analysis, assessing associations with clinicopathological characteristics, GSEA and RT-qPCR verification, five lncRNAs (LINC00501, LINC00365, SOX21-AS1, GK-IT1 and DLEU7-AS1) were identified that may be functionally associated with GC development, and thus may be used as novel diagnostic biomarkers for patients with GC.

In the present study, five lncRNAs (LINC00501, LINC00365, SOX21-AS1, GK-IT1 and DLEU7-AS1), 29 miRNAs and 114 mRNAs were incorporated into a ceRNA network. Among the included lncRNAs, LINC00501 and LINC00365 were associated with the most miRNAs, suggesting that they may be key regulators of GC due to their ceRNA functionality. Previous studies have demonstrated that a number of the selected mRNAs and predicted miRNAs, including miR-301b-3p (31), miR-4295 (32), miR-206 (33), miR-613 (34), miR-429 (35), forkhead box $\mathrm{C} 1$ (36), G protein-coupled receptor class $\mathrm{C}$ group 5 member A (37) and kinesin family member 23 (38), are 
Table III. Specific lncRNAs that target specific miRNAs.

lncRNAs

miRNAs

LINC00501

miR-301b-3p, miR-4295, miR-3666, miR-206, miR-613, miR-429, miR-23b-3p, miR-24-3p, miR-363-3p, miR-338-3p, miR-455-5p, miR-129-5p, miR-490-3p

LINC00365 miR-17-5p, miR-20b-5p, miR-429, miR-590-5p, miR-761, miR-3619-5p, miR-216b-5p, miR-363-3p, miR-338-3p, miR-449c-5p, miR-125a-5p, miR-125b-5p, miR-129-5p

SOX21-AS1 miR-301b-3p, miR-4295, miR-3666, miR-212-3p, miR-761, miR-3619-5p, miR-107, miR-338-3p, miR-125a-5p, miR-125b-5p, miR-455-5p, miR-129-5p

GK-IT1 miR-4770, miR-24-3p, miR-129-5p

DLEU7-AS1 miR-507, miR-142-3p, miR-761, miR-3619-5p, miR-23b-3p, miR-27a-3p, miR-107, miR-338-3p, miR-375

lncRNA, long non-coding RNA; miRNA/miR, microRNA; LINC00, long intergenic non-protein coding RNA; DLEU7-AS1, DLEU7 antisense RNA 1; GK-IT1, GK intronic transcript 1; SOX21-AS1, SOX21 antisense divergent transcript 1.

Table IV. Specific miRNAs that target specific mRNAs.

\begin{tabular}{|c|c|}
\hline miRNAs & mRNAs \\
\hline miR-107 & CYP2C8, DEPDC1B, FOXC1, CREBRF, GPRC5A, SYNDIG1, SERPINB5, KIF23, RPS6KB1, \\
\hline \multirow[t]{2}{*}{$\operatorname{miR}-125 a-5 p$} & SWSAP1, UBE2J1, SWSAP1, UBE2J1, AGTR1, DMRTA2, GK, ADHFE1, ITIH5, RPS6KB1, \\
\hline & CAMK2N1, REP15, NALCN, PDE3A, CKAP2L, GIF, NEUROD2, FANCI, PRKCB, ZIC2 \\
\hline miR-142-3p & ADAMTS3, CDC6, FLVCR1 \\
\hline \multirow[t]{3}{*}{ miR-17-5p } & PRKCB, KIF23, MSTO1, TMEM100, NAA50, LPGAT1, PBK, SYT16, AUNIP, ATP1B3, \\
\hline & RHOV, BAMBI, MUC17, MMP3, UBE2J1, AADAC, RND3, STIL, NETO2, KIF26B, FOXC1, \\
\hline & IGFBP5, PON2, TGM2, FAM83D, NMUR2 \\
\hline $\operatorname{miR}-206$ & RSPO3, SLC2A12, NETO2, PABPC1L2B, NALCN, DGKH \\
\hline $\operatorname{miR}-20 b-5 p$ & NETO2, KIF26B, KIF23, TMEM100, LPGAT1, BAMBI, PON2, UBE2J1 \\
\hline miR-212-3p & SOWAHA, TLN2, OCLN, MUC13, CCNB1, DUSP9 \\
\hline $\operatorname{miR}-216 b-5 p$ & ANKRD22, ZIC5, FADS1, SOX11, RND3, ANXA10, TRAM1L1 \\
\hline $\operatorname{miR}-23 b-3 p$ & VSNL1, NAA50, LPGAT1, ALDH1A2, ZIC5, CA2 \\
\hline $\operatorname{miR}-24-3 p$ & $\begin{array}{l}\text { CCNB1, RSPO3, FSCN1, SULT2A1, CDX2, CD3D, STIL, NETO2, CDKN2A, DSC2, TLN2, } \\
\text { OSMR }\end{array}$ \\
\hline $\operatorname{miR}-27 a-3 p$ & $\begin{array}{l}\text { GRIN2D, PRKCB, HRK, RPS6KB1, CREBRF, WISP1, RND3, HOXA10, PAPSS2, NAA50, } \\
\text { SOWAHA, DEPDC1B, ONECUT2 }\end{array}$ \\
\hline $\operatorname{miR}-301 b-3 p$ & GDA, LGALSL, ZIC5, IGFBP5, NAA50 \\
\hline miR-338-3p & ONECUT2, GPRC5A, LGALSL, SERPINA5, CEACAM6 \\
\hline $\operatorname{miR}-3619-5 p$ & NAA50, LPGAT1, PLCB2, P2RX6, SCAMP4, LTF, NRG4, FSCN1 \\
\hline $\operatorname{miR}-363-3 p$ & $\begin{array}{l}\text { FZD10, OSMR, DSC2, KLHL29, SYNDIG1, PAPSS2, SOX11, TRIP13, ZIC5, SOSTDC1, } \\
\text { SYNPO2 }\end{array}$ \\
\hline $\operatorname{miR}-3666$ & NAA50 \\
\hline $\operatorname{miR}-375$ & NETO2, CLDN1, TGM2, MOSPD3 \\
\hline $\operatorname{miR}-429$ & ADAMTS3, NOG, RND3, OCLN, TRAM1L1, NALCN, FLVCR1, FSCN1, KLHL29, PRKCB, \\
\hline & ONECUT2, KCTD8, TLN2, RPS6KB1, PMAIP1, KYNU \\
\hline $\operatorname{miR}-4295$ & NAA50 \\
\hline $\operatorname{miR}-449 c-5 p$ & $\begin{array}{l}\text { PAPSS2, ACADL, NMUR2, TRIM73, ATP1B3, CARNS1, MLANA, ACAD8, SERPINB5, } \\
\text { KLHL29, NETO2, RPS6KB1, DEPDC1B, SOX11, AGR3 }\end{array}$ \\
\hline $\operatorname{miR}-4770$ & IGFBP5 \\
\hline $\operatorname{miR}-490-3 p$ & SOX11, GCNT3 \\
\hline miR-507 & DGKH, DSC2, RPS6KB1, LPGAT1, WISP1, RABGEF1 \\
\hline $\operatorname{miR}-590-5 p$ & FBXL13, VSNL1, IL1B, ALDH1A1 \\
\hline $\operatorname{miR}-613$ & DGKH, RSPO3, NALCN, NETO2, PABPC1L2B, SLC2A12 \\
\hline miR-761 & SCAMP4, NAA50, FSCN1, P2RX6, NRG4, LTF, LPGAT1 \\
\hline
\end{tabular}

miRNA/miR, microRNA. 
A Bar chart of Biological Process categories

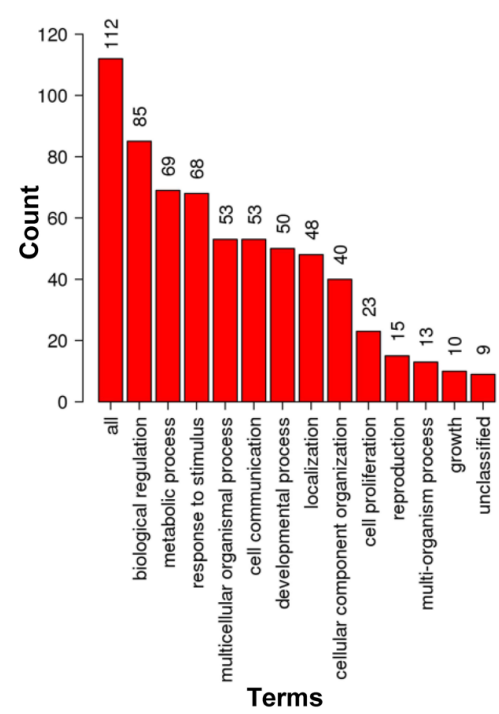

B

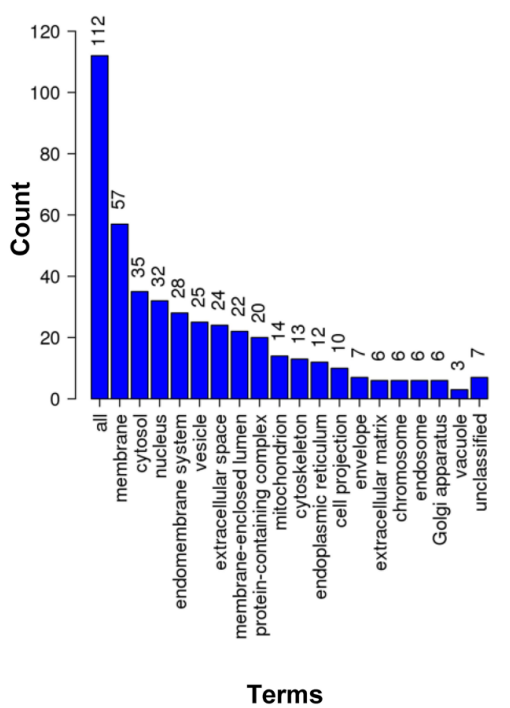

C Bar chart of Molecular Function categories

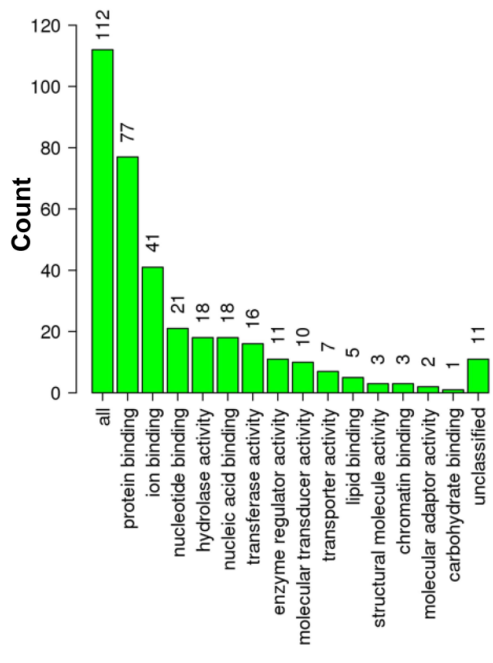

Terms

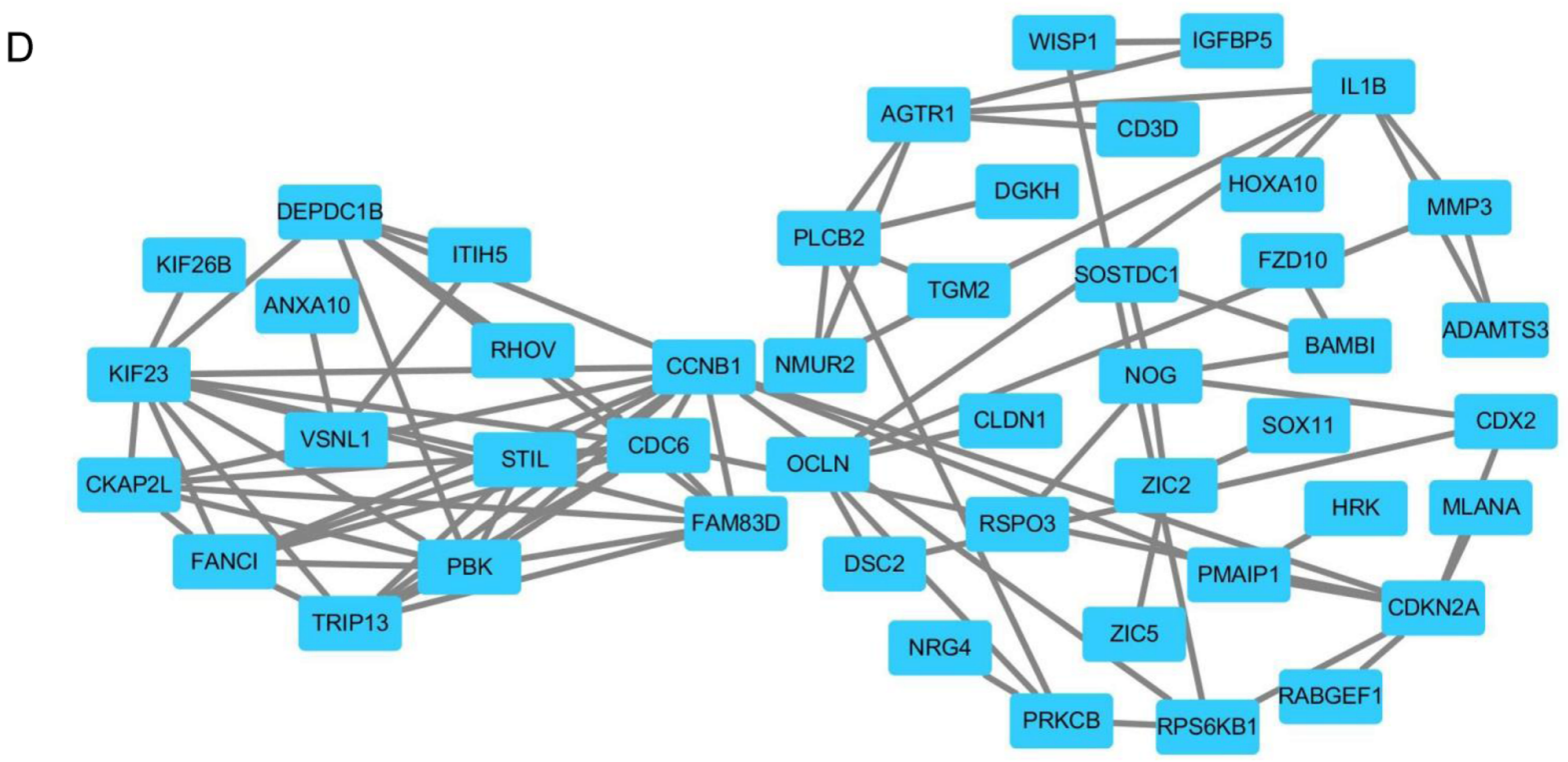

Figure 4. Functional enrichment analysis of differentially expressed genes. Gene Ontology analysis of significantly enriched (A) biological processes, (B) cellular components and (C) molecular functions. (D) Protein-protein interaction network.

associated with GC. GO analysis revealed that the 114 mRNAs within this network may be involved in biological processes, including 'biological regulation', 'metabolic process', 'response to stimulus', 'multicellular organismal process', 'cell communication', 'developmental process', 'localization', 'cellular component organization', 'cell proliferation', 'reproduction' and 'multi-organism process growth'. The mRNAs were primarily enriched for cellular component terms, including 'membrane', 'cytosol', 'nucleus', 'endomembrane system', 'vesicle', 'extracellular space', 'membrane-enclosed lumen' and 'protein-containing complex'. For molecular functions terms, the mRNAs were concentrated in 'protein binding', 'ion binding', 'nucleotide binding', 'hydrolase activity', 'nucleic acid binding', 'transferase activity', 'enzyme regulator activity', 'molecular transducer activity', ' transporter activity' and 'lipid binding'. PPI analyses revealed that these IncRNAs may promote or inhibit the development of GC by targeting a range of cell cycle-related mRNAs, such as CCNB1 (39,40), FAM83D (41) and CDC6 (42).
The ceRNA hypothesis posits that IncRNAs can competitively bind miRNAs, thereby altering their ability to influence mRNA translation (8). Recent studies have demonstrated that ceRNAs play important functional roles in GC $(43,44)$. In the present study, a ceRNA network was constructed, GO and PPI analyses were performed to elucidate the mechanisms regarding how these lncRNAs exert their functions. The results suggest LINC00501 may be involved in cell proliferation by targeting mRNA fascin actin-bundling protein 1 (FSCN1) via miR-429. Notably, a previous study demonstrated that miR-429 acts as a tumor suppressor by targeting FSCN1 in GC (45), which was consistent with the results of the present study. The results of the present study also suggest that LINC00365 may be involved in the cell cycle by targeting mRNA FAM83D via miR-17-5p. In addition, SOX21-AS1 and GK-IT1 may be involved in the cell cycle by targeting mRNA CCNB1 via miR-212-3p and miR-24-3p, respectively. Furthermore, DLEU7-AS1 may play a role in carcinogenesis by regulating the cell cycle through 

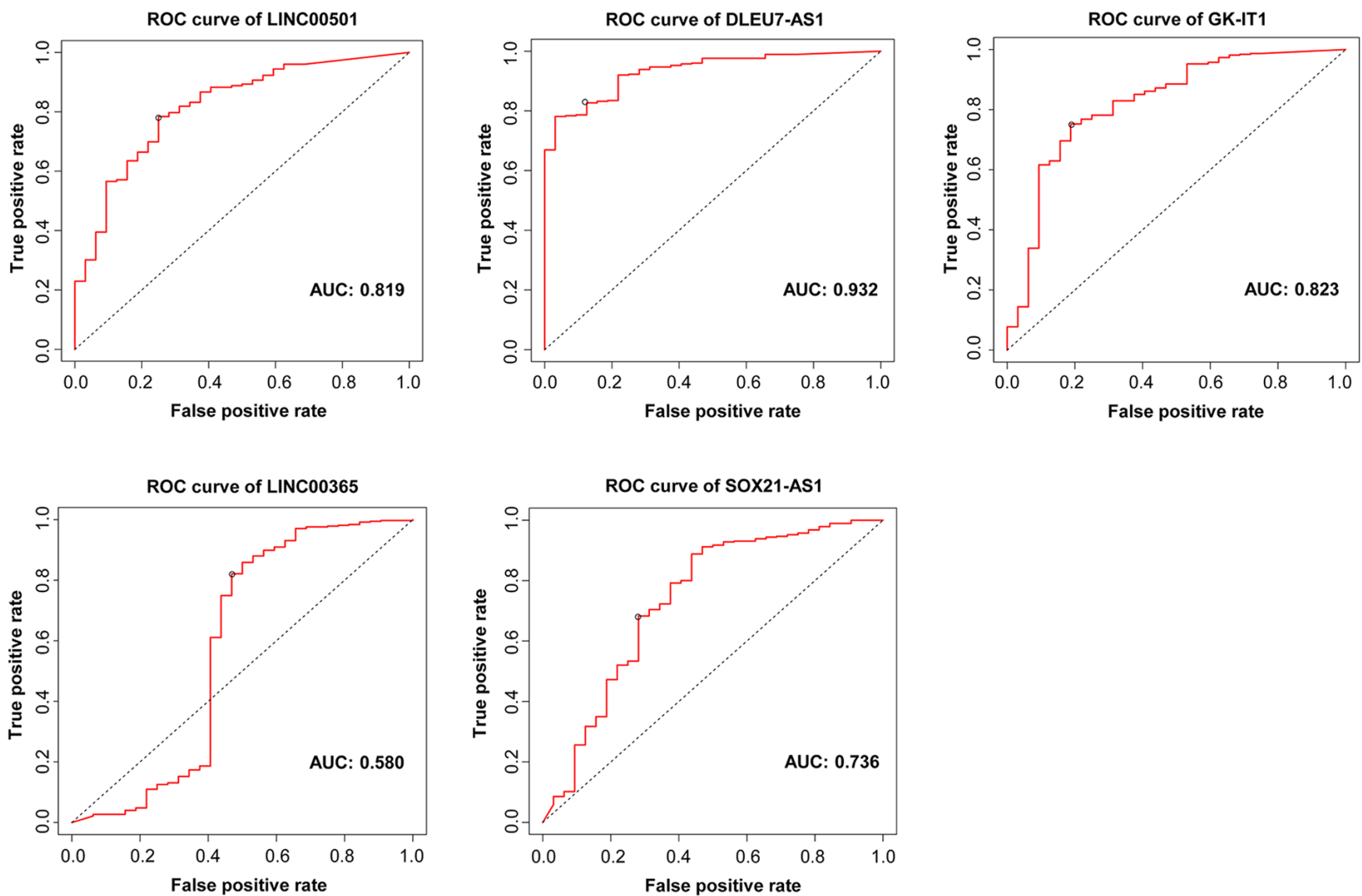

Figure 5. ROC curve analysis of the five long non-coding RNAs. The red line represents the sensitivity curve, while the black line represents the identifying line. ROC, receiver operating characteristic; AUC, area under the curve; LINC00, long intergenic non-protein coding RNA; SOX21-AS1, SOX21 antisense divergent transcript 1; GK-IT1, GK intronic transcript 1; DLEU7-AS1, DLEU7 antisense RNA 1.
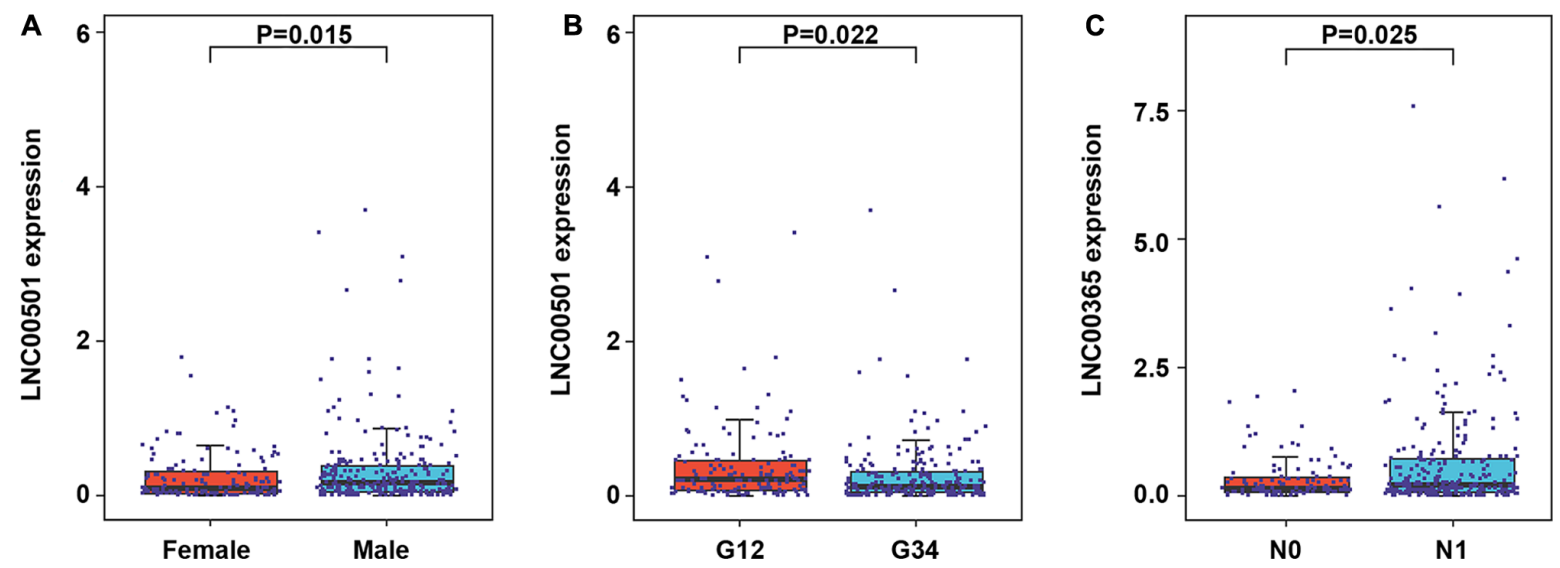

Figure 6. Associations between long non-coding RNAs and clinicopathological characteristics. Association between LINC00501 expression and (A) sex and (B) tumor grade. (C) Association between LINC00365 expression and lymph node metastasis. LINC00, long intergenic non-protein coding RNA.

miR-142-3p/CDC6. In the present study, ROC analysis revealed that AUC values for LINC00501, LINC00365, SOX21-AS1, GK-IT1 and DLEU7-AS1 were all $>0.50$, which suggests that these five lncRNAs have the potential to serve as valuable diagnostic biomarkers for GC. Notably, analysis of the associations with clinicopathological characteristics revealed that LINC00501 expression was associated with sex and tumor grade. Furthermore, LINC00365 expression was upregulated in N1 stage samples compared with that in N0 stage samples, suggesting that LINC00365 may be an indicator for early stage GC diagnosis without lymph node metastasis. GSEA of the three IncRNAs indicated that LINC00501 may modulate GC progression via the TGF-beta, MAPK, WNT and mTOR signaling pathways, LINC00365 via MAPK signaling and SOX21-AS1 via the cell cycle. Several studies have highlighted the important roles of TGF-beta signaling pathway (46), MAPK signaling pathway (47), WNT signaling pathway (48) and the cell cycle (49) in GC. 
A

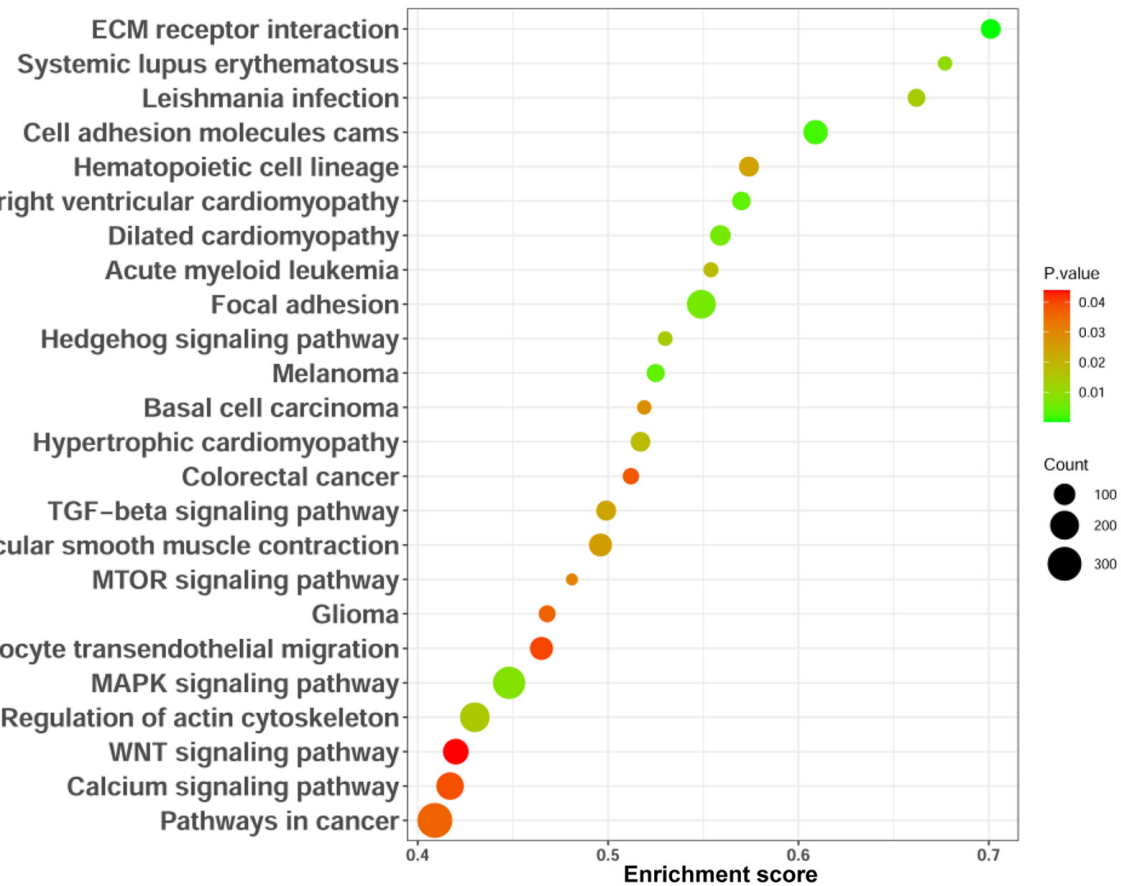

B

Systemic lupus erythematosus

Leishmania infection

ECM receptor interaction

Cell adhesion molecules cams

Focal adhesion

Antigen processing and presentation-

Viral myocarditis

Dilated cardiomyopathy

Vascular smooth muscle contraction

Basal cell carcinoma

Melanoma

Arrhythmogenic right ventricular cardiomyopathy

Pathogenic escherichia coli infection

Progesterone mediated oocyte maturation

Leukocyte transendothelial migration

Gap junction

Regulation of actin cytoskeleton

Cytokine cytokine receptor interaction

Pathways in cancer-

MAPK signaling pathway

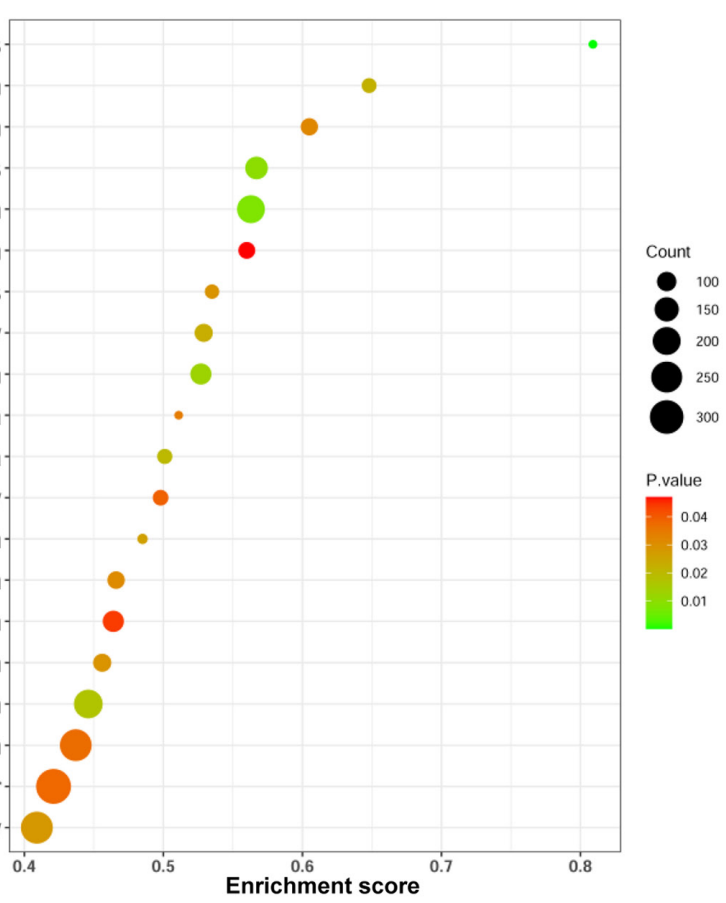

C

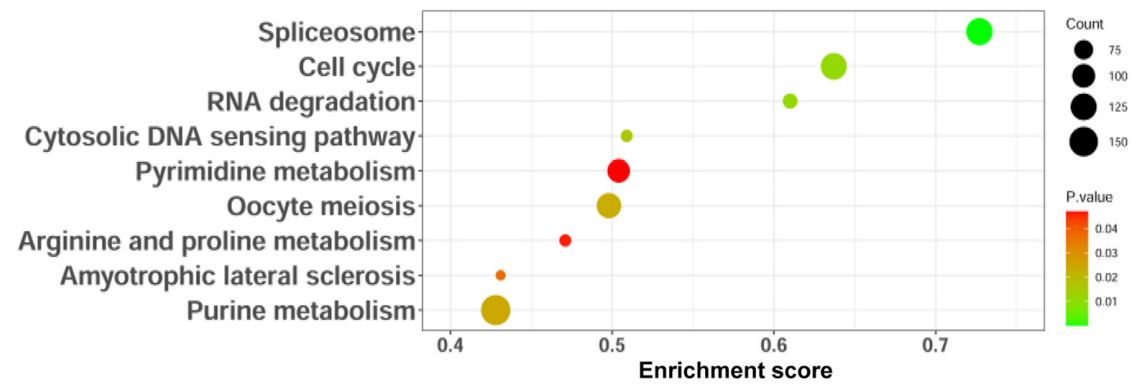

Figure 7. GSEA pathways of the long non-coding RNAs. GSEA pathways of (A) LINC00501, (B) LINC00365 and (C) SOX21-AS1. GSEA, gene set enrichment analysis; LINC00, long intergenic non-protein coding RNA; SOX21-AS1, SOX21 antisense divergent transcript 1.

LINC00501 has been identified as a prognostic factor associated with the overall survival of patients with hepatocellular carcinoma (HCC) (50-52). LINC00365 influences the
Wnt/ $\beta$-catenin signaling pathway, which modulates colorectal cancer (CRC) progression (53), and breast cancer cell viability may be regulated by the LINC00365-secretoglobin 

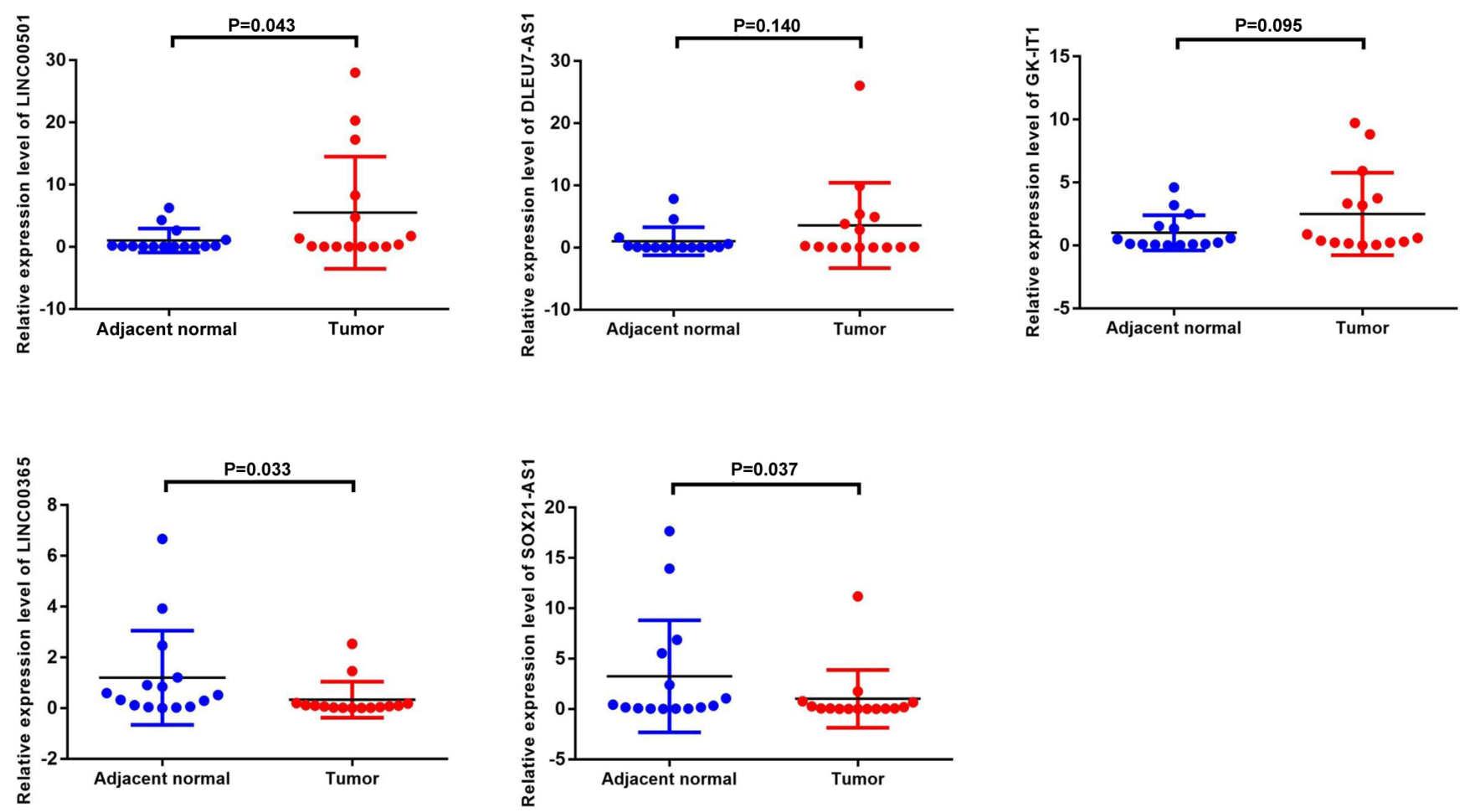

Figure 8. Reverse transcription-quantitative PCR-based validation of the expression levels of five long non-coding RNAs in gastric cancer tissues. LINC00, long intergenic non-protein coding RNA; DLEU7-AS1, DLEU7 antisense RNA 1; GK-IT1, GK intronic transcript 1; SOX21-AS1, SOX21 antisense divergent transcript 1 .

family 2A member 1 (SCGB2A1) axis, which targets NF- $\kappa \mathrm{B}$ signaling (54). In GC, the expression levels of LINC00365 and SCGB2A1 are downregulated in tumor tissues, which is associated with a shorter survival time (55). Notably, this LINC00365/SCGB2A1 axis and associated NF- $\kappa B$ suppression are associated with GC progression (55). In the present study, SOX21-AS1 was demonstrated to be associated with GC in the ceRNA network. Previous studies have reported that SOX21-AS1 is associated with different types of cancer, including cervical cancer $(56,57)$, HCC $(58)$, lung adenocarcinoma (59), CRC (60) and oral cancer (61). Previous studies have demonstrated that SOX21-AS1 can function as a ceRNA to sequester miRNAs, which in turn modulates gene expression $(57,60)$. For example, SOX21-AS1 participates in cervical cancer progression by competitively binding miR-7/voltage dependent anion channel 1 (57), while it sequesters miR-145 in $\mathrm{CRC}$, which promotes tumor progression via the enhanced expression of myosin VI (60). SOX21-AS1 knockdown in nephroblastoma cells in vitro disrupts the proliferation and colony formation of these cells through a mechanism associated with p57 upregulation, and results in cell cycle arrest (62). In addition, SOX21-AS1 is associated with HCC progression and patient prognosis via a mechanism associated with $\mathrm{p} 21$ epigenetic silencing (58).

Although the differences in DLEU7-AS1 and GK-IT1 expression were not significant, they exhibited an upward trend consistent with the present bioinformatics analyses. This lack of significance may be attributed to the limited sample size in the present study. Thus, future studies with larger sample sizes are required to confirm the results presented here. A previous study demonstrated that DLEU7-AS1 expression is upregulated in CRC, and is associated with CRC stage, metastasis and poor patient prognosis (63). From a mechanistic perspective, DLEU7-AS1 is considered to modulate the Wnt/ $\beta$-catenin signaling pathway in CRC cells, which influences the ability of these cells to proliferate and invade proximal or distal tissues (63). GK-IT1 has been reported to be positively associated with overall survival in patients with esophageal adenocarcinoma (64).

Several studies have investigated ceRNA networks for $\mathrm{GC}$, which have aimed to screen lncRNAs involved in GC via analysis of gene expression data obtained from TCGA database (65-67). Different original non-coding RNA microarray data were used for the analysis, thus different lncRNAs were identified as biomarkers for GC. The present study aimed to screen novel lncRNAs involved in GC via analysis of gene expression data obtained from both the GEO and TCGA databases, which makes the results more reliable and repeatable. However, the present study is not without limitations. Although the expression levels of the five lncRNAs were verified in GC tissues, the lack of validation of these biomarkers in serum is a major limitation of the present study. Furthermore, future studies are required to confirm the functions of the identified lncRNAs in GC.

In conclusion, integrated analysis of TCGA and GEO datasets and a series of analyses identified five differentially expressed lncRNAs (LINC00501, LINC00365, SOX21-AS1, GK-IT1 and DLEU7-AS1), which may be used as novel diagnostic biomarkers associated with GC, and may be functionally associated with GC development and progression. Thus, the present study provides novel insight into the mechanistic basis and biological functions of lncRNAs in GC. 


\section{Acknowledgements}

Not applicable.

\section{Funding}

The present study was funded by Key Research and Development of Social and People's Livelihood (grant no. cstc2018jscx-mszdX0031) and the Natural Science Foundation of Chongqing in China (grant no. cstc2019jcyj-msxmX0259).

\section{Availability of data and materials}

The datasets used and/or analyzed during the current study are available from the corresponding author upon reasonable request.

\section{Authors' contributions}

YJ, XZ and FS conceived the present study. YJ and XZ participated in experiment design and coordination. YJ drafted and revised the manuscript for important intellectual content, and performed reverse transcription-quantitative PCR analysis. LR, JS and WZ collected the tissue samples. YH, MH, YX and YL performed statistical analysis and revised the manuscript for important intellectual content. YJ and FS confirmed the authenticity of all the raw data. All authors have read and approved the final manuscript.

\section{Ethics approval and consent to participate}

The present study was approved by the Research Ethics Committee of the First Affiliated Hospital of Chongqing Medical University (Chongqing, China; approval no. 2016140) and written informed consent was provided by all patients prior to the study start.

\section{Patient consent for publication}

Not applicable.

\section{Competing interests}

The authors declare that they have no competing interests.

\section{References}

1. Bray F, Ferlay J, Soerjomataram I, Siegel RL, Torre LA and Jemal A: Global cancer statistics 2018: GLOBOCAN estimates of incidence and mortality worldwide for 36 cancers in 185 countries. CA Cancer J Clin 68: 394-424, 2018.

2. Forrest ME and Khalil AM: Review: Regulation of the cancer epigenome by long non-coding RNAs. Cancer Lett 407: 106-112, 2017.

3. Ponting CP, Oliver PL and Reik W: Evolution and functions of long noncoding RNAs. Cell 136: 629-641, 2009.

4. Cabili MN, Trapnell C, Goff L, Koziol M, Tazon-Vega B, Regev A and Rinn JL: Integrative annotation of human large intergenic noncoding RNAs reveals global properties and specific subclasses. Genes Dev 25: 1915-1927, 2011.

5. Wang CJ, Zhu CC, Xu J, Wang M, Zhao WY, Liu Q, Zhao G and Zhang ZZ: The lncRNA UCA1 promotes proliferation, migration, immune escape and inhibits apoptosis in gastric cancer by sponging anti-tumor miRNAs. Mol Cancer 18: 115, 2019.
6. Deng W, Zhang Y, Cai J, Zhang J, Liu X, Yin J, Bai Z, Yao H and Zhang Z: IncRNA-ANRIL promotes gastric cancer progression by enhancing NF-kB signaling. Exp Biol Med (Maywood) 244: 953-959, 2019

7. Ba MC, Ba Z, Long H, Cui SZ, Gong YF, Yan ZF, Lin KP, Wu YB and Tu YN: lncRNA AC093818.1 accelerates gastric cancer metastasis by epigenetically promoting PDK1 expression. Cell Death Dis 11: 64, 2020.

8. Russo F, Fiscon G, Conte F, Rizzo M, Paci P and Pellegrini M: Interplay between long noncoding RNAs and MicroRNAs in cancer. Methods Mol Biol 1819: 75-92, 2018.

9. Liu HT, Ma RR, Lv BB, Zhang H, Shi DB, Guo XY, Zhang GH and Gao P: lncRNA-HNF1A-AS1 functions as a competing endogenous RNA to activate PI3K/AKT signalling pathway by sponging miR-30b-3p in gastric cancer. Br J Cancer 122 : $1825-1836,2020$

10. Su YZ, Cui MF, Du J and Song B: lncRNA DCST1-AS1 regulated cell proliferation, migration, invasion and apoptosis in gastric cancer by targeting miR-605-3p. Eur Rev Med Pharmacol Sci 24: 1158-1167, 2020.

11. Zhang G, Li S, Lu J, Ge Y, Wang Q, Ma G, Zhao Q, Wu D, Gong W, Du M, et al: lncRNA MT1JP functions as a ceRNA in regulating FBXW7 through competitively binding to miR-92a-3p in gastric cancer. Mol Cancer 17: 87, 2018.

12. Luan M, Shi SS, Shi DB, Liu HT, Ma RR, Xu XQ, Sun YJ and Gao P: TIPRL, a Novel tumor suppressor, suppresses cell migration, and invasion through regulating AMPK/mTOR Signaling pathway in gastric cancer. Front Oncol 10: 1062, 2020.

13. Song YX, Sun JX, Zhao JH, Yang YC, Shi JX, Wu ZH, Chen XW, Gao P, Miao ZF and Wang ZN: Non-coding RNAs participate in the regulatory network of CLDN4 via ceRNA mediated miRNA evasion. Nat Commun 8: 289, 2017.

14. Barrett T, Wilhite SE, Ledoux P, Evangelista C, Kim IF, Tomashevsky M, Marshall KA, Phillippy KH, Sherman PM, Holko M, et al: NCBI GEO: Archive for functional genomics data sets-update. Nucleic Acids Res 41 (Database Issue): D991-D995, 2013.

15. Liu J, Lichtenberg T, Hoadley KA, Poisson LM, Lazar AJ, Cherniack AD, Kovatich AJ, Benz CC, Levine DA, Lee AV, et al: An integrated TCGA Pan-cancer clinical data resource to drive high-quality survival outcome analytics. Cell 173: 400-416.e411, 2018.

16. Leek JT, Johnson WE, Parker HS, Jaffe AE and Storey JD: The sva package for removing batch effects and other unwanted variation in high-throughput experiments. Bioinformatics 28: 882-883, 2012.

17. Ritchie ME, Phipson B, Wu D, Hu Y, Law CW, Shi W and Smyth GK: limma powers differential expression analyses for RNA-sequencing and microarray studies. Nucleic Acids Res 43: e47, 2015 .

18. Jeggari A, Marks DS and Larsson E: miRcode: A map of putative microRNA target sites in the long non-coding transcriptome. Bioinformatics 28: 2062-2063, 2012.

19. Agarwal V, Bell GW, Nam JW and Bartel DP: Predicting effective microRNA target sites in mammalian mRNAs. Elife 4: e05005, 2015.

20. Chou CH, Shrestha S, Yang CD, Chang NW, Lin YL, Liao KW, Huang WC, Sun TH, Tu SJ, Lee WH, et al: miRTarBase update 2018: A resource for experimentally validated microRNA-target interactions. Nucleic Acids Res 46: D296-D302, 2018.

21. Shannon P, Markiel A, Ozier O, Baliga NS, Wang JT, Ramage D, Amin N, Schwikowski B and Ideker T: Cytoscape: A software environment for integrated models of biomolecular interaction networks. Genome Res 13: 2498-2504, 2003.

22. Liao Y, Wang J, Jaehnig EJ, Shi Z and Zhang B: WebGestalt 2019: Gene set analysis toolkit with revamped UIs and APIs. Nucleic Acids Res 47: W199-W205, 2019.

23. Szklarczyk D, Franceschini A, Wyder S, Forslund K, Heller D, Huerta-Cepas J, Simonovic M, Roth A, Santos A, Tsafou KP, et al: STRING v10: Protein-protein interaction networks, integrated over the tree of life. Nucleic Acids Res 43 (Database Issue): D447-D452, 2015.

24. Martin IG, Dixon MF, Sue-Ling H, Axon AT and Johnston D: Goseki histological grading of gastric cancer is an important predictor of outcome. Gut 35: 758-763, 1994.

25. Washington $\mathrm{K}: 7$ th edition of the AJCC cancer staging manual: Stomach. Ann Surg Oncol 17: 3077-3079, 2010.

26. Livak KJ and Schmittgen TD: Analysis of relative gene expression data using real-time quantitative PCR and the 2(-Delta Delta C(T)) method. Methods 25: 402-408, 2001. 
27. Lei S, He Z, Chen T, Guo X, Zeng Z, Shen Y and Jiang J: Long noncoding RNA 00976 promotes pancreatic cancer progression through OTUD7B by sponging miR-137 involving EGFR/MAPK pathway. J Exp Clin Cancer Res 38: 470, 2019.

28. Lu W, Zhang H, Niu Y, Wu Y, Sun W, Li H, Kong J, Ding K, Shen $\mathrm{HM}, \mathrm{Wu} \mathrm{H}$, et al: Long non-coding RNA linc00673 regulated non-small cell lung cancer proliferation, migration, invasion and epithelial mesenchymal transition by sponging miR-150-5p. Mol Cancer 16: 118, 2017.

29. Wei GH and Wang X: lncRNA MEG3 inhibit proliferation and metastasis of gastric cancer via p53 signaling pathway. Eur Rey Med Pharmacol Sci 21: 3850-3856, 2017.

30. Yao N, Fu Y, Chen L, Liu Z, He J, Zhu Y, Xia T and Wang S: Long non-coding RNA NONHSAT101069 promotes epirubicin resistance, migration, and invasion of breast cancer cells through NONHSAT101069/miR-129-5p/Twist1 axis. Oncogene 38: 7216-7233, 2019

31. Fan H, Jin X, Liao C, Qiao L and Zhao W: MicroRNA-301b-3p accelerates the growth of gastric cancer cells by targeting zinc finger and BTB domain containing 4. Pathol Res Pract 215: 152667, 2019.

32. Yan R, Li K, Yuan DW, Wang HN, Zhang Y, Dang CX and Zhu K: Downregulation of microRNA-4295 enhances cisplatin-induced gastric cancer cell apoptosis through the EGFR/PI3K/Akt signaling pathway by targeting LRIG1. Int J Oncol 53: 2566-2578, 2018.

33. Deng M, Qin Y, Chen X, Wang Q and Wang J: MiR-206 inhibits proliferation, migration, and invasion of gastric cancer cells by targeting the MUC1 gene. Onco Targets Ther 12: 849-859, 2019.

34. Lu Y, Tang L, Zhang Q, Zhang Z and Wei W: MicroRNA-613 inhibits the progression of gastric cancer by targeting CDK9. Artif Cells Nanomed Biotechnol 46: 980-984, 2018

35. Sun T, Wang C, Xing J and Wu D: miR-429 modulates the expression of c-myc in human gastric carcinoma cells. Eur J Cancer 47: $2552-2559,2011$.

36. Xu Y, Shao QS, Yao HB, Jin Y, Ma YY and Jia LH: Overexpression of FOXC1 correlates with poor prognosis in gastric cancer patients. Histopathology 64: 963-970, 2014.

37. Liu H, Zhang Y, Hao X, Kong F, Li X, Yu J and Jia Y: GPRC5A overexpression predicted advanced biological behaviors and poor prognosis in patients with gastric cancer. Tumour Biol 37: 503-510, 2016

38. Liu Y, Chen H, Dong P, Xie G, Zhou Y, Ma Y, Yuan X, Yang J, Han L, Chen L and Shen L: KIF23 activated Wnt/ $\beta$-catenin signaling pathway through direct interaction with Amer1 in gastric cancer. Aging (Albany NY) 12: 8372-8396, 2020.

39. Chen EB, Qin X, Peng K, Li Q, Tang C, Wei YC, Yu S, Gan L and Liu TS: HnRNPR-CCNB1/CENPF axis contributes to gastric cancer proliferation and metastasis. Aging (Albany NY) 11 7473-7491, 2019.

40. Ding L, Yang L, He Y, Zhu B, Ren F, Fan X, Wang Y, Li M, Li J, Kuang Y, et al: CREPT/RPRD1B associates with Aurora B to regulate Cyclin B1 expression for accelerating the $\mathrm{G} 2 / \mathrm{M}$ transition in gastric cancer. Cell Death Dis 9: 1172, 2018.

41. Huang M, Ma X, Shi H, Hu L, Fan Z, Pang L, Zhu F, Yang X, $\mathrm{Xu}$ W, Liu B, et al: FAM83D, a microtubule-associated protein, promotes tumor growth and progression of human gastric cancer. Oncotarget 8: 74479-74493, 2017.

42. Zhao B, Zhang J, Chen X, Xu H and Huang B: Mir-26b inhibits growth and resistance to paclitaxel chemotherapy by silencing the CDC6 gene in gastric cancer. Arch Med Sci 15: 498-503, 2019.

43. Cao Y, Xiong JB, Zhang GY, Liu Y, Jie ZG and Li ZR: Long Noncoding RNA UCA1 Regulates PRL-3 expression by sponging MicroRNA-495 to promote the progression of gastric cancer. Mol Ther Nucleic Acids 19: 853-864, 2020.

44. Cui Y, Pu R, Ye J, Huang H, Liao D, Yang Y, Chen W, Yao Y and $\mathrm{He} \mathrm{Y}$ : lncRNA FAM230B promotes gastric cancer growth and metastasis by regulating the miR-27a-5p/TOP2A Axis. Dig Dis Sci: Sep 10, 2020 (Epub ahead of print). doi: 10.1007/s10620-020-06581-z.

45. Zhang M, Dong BB, Lu M, Zheng MJ, Chen H, Ding JZ, Xu AM and $\mathrm{Xu}$ YH: miR-429 functions as a tumor suppressor by targeting FSCN1 in gastric cancer cells. Onco Targets Ther 9: 1123-1133, 2016

46. Chen ZL, Qin L, Peng XB, Hu Y and Liu B: INHBA gene silencing inhibits gastric cancer cell migration and invasion by impeding activation of the TGF- $\beta$ signaling pathway. J Cell Physiol 234: 18065-18074, 2019.

47. Xiang Z, Li J, Song S, Wang J, Cai W, Hu W, Ji J, Zhu Z, Zang L, Yan R and Yu Y: A positive feedback between IDO1 metabolite and COL12A1 via MAPK pathway to promote gastric cancer metastasis. J Exp Clin Cancer Res 38: 314, 2019.
48. Gao J, Zhao C, Liu Q, Hou X, Li S, Xing X, Yang C and Luo Y: Cyclin G2 suppresses Wnt/ $\beta$-catenin signaling and inhibits gastric cancer cell growth and migration through Dapperl. J Exp Clin Cancer Res 37: 317, 2018.

49. Zhang L, Kang W, Lu X, Ma S, Dong L and Zou B: IncRNA CASC11 promoted gastric cancer cell proliferation, migration and invasion in vitro by regulating cell cycle pathway. Cell Cycle 17: 1886-1900, 2018

50. Hou Y, Yu Z, Tam NL, Huang S, Sun C, Wang R, Zhang X, Wang Z, Ma Y, He X and Wu L: Exosome-related lncRNAs as predictors of HCC patient survival: A prognostic model. Am J Transl Res 10: 1648-1662, 2018.

51. Lou X, Li J, Yu D, Wei YQ, Feng S and Sun JJ: Comprehensive analysis of five long noncoding RNAs expression as competing endogenous RNAs in regulating hepatoma carcinoma. Cancer Med 8: 5735-5749, 2019

52. Ye J, Zhang J, Lv Y, Wei J, Shen X, Huang J, Wu S and Luo X: Integrated analysis of a competing endogenous RNA network reveals key long noncoding RNAs as potential prognostic biomarkers for hepatocellular carcinoma. J Cell Biochem 120: 13810-13825, 2019.

53. Zhu Y, Bian Y, Zhang Q, Hu J, Li L, Yang M, Qian H, Yu L, Liu B and Qian X: LINC00365 promotes colorectal cancer cell progression through the Wnt/ $\beta$-catenin signaling pathway. J Cell Biochem 121: 1260-1272, 2020.

54. Zhang L, Yan X, Yu S, Zhong X, Tian R, Xu L, Bian X and Su J: LINC00365-SCGB2A1 axis inhibits the viability of breast cancer through targeting NF- $\kappa$ B signaling. Oncol Lett 19: 753-762, 2020.

55. Yan XY, Zhang JJ, Zhong XR, Yu SH, Xu L, Tian R, Sun LK and Su J: The LINC00365/SCGB2A1 (Mammaglobin B) axis down-regulates $\mathrm{NF}-\kappa \mathrm{B}$ signaling and is associated with the progression of gastric cancer. Cancer Manag Res 12: 621-631, 2020

56. Wang R, Li Y,Du P,Zhang X,Li X and Cheng G: Hypomethylation of the lncRNA SOX21-AS1 has clinical prognostic value in cervical cancer. Life Sci 233: 116708, 2019.

57. Zhang X, Zhao X, Li Y, Zhou Y and Zhang Z: Long noncoding RNA SOX21-AS1 promotes cervical cancer progression by competitively sponging miR-7/VDAC1. J Cell Physiol 234: 17494-17504, 2019.

58. Wei C, Wang H, Xu F, Liu Z and Jiang R: lncRNA SOX21-AS1 is associated with progression of hepatocellular carcinoma and predicts prognosis through epigenetically silencing $\mathrm{p} 21$. Biomed Pharmacother 104: 137-144, 2018

59. Lu X, Huang C, He X, Liu X, Ji J, Zhang E, Wang W and Guo R: A Novel long non-coding RNA, SOX21-AS1, indicates a poor prognosis and promotes lung adenocarcinoma proliferation. Cell Physiol Biochem 42: 1857-1869, 2017.

60. Wei AW and Li LF: Long non-coding RNA SOX21-AS1 sponges miR-145 to promote the tumorigenesis of colorectal cancer by targeting MYO6. Biomed Pharmacother 96: 953-959, 2017.

61. Yang CM, Wang TH, Chen HC, Li SC, Lee MC, Liou HH, Liu PF, Tseng YK, Shiue YL, Ger LP and Tsai KW: Aberrant DNA hypermethylation-silenced SOX21-AS1 gene expression and its clinical importance in oral cancer. Clin Epigenetics 8: 129,2016

62. Zhang J, Hou T, Qi X, Wang J and Sun X: SOX21-AS1 is associated with clinical stage and regulates cell proliferation in nephroblastoma. Biosci Rep 39: BSR20190602, 2019.

63. Liu XB, Han C and Sun CZ: Long non-coding RNA DLEU7-AS1 promotes the occurrence and development of colorectal cancer via Wnt/ $\beta$-catenin pathway. Eur Rev Med Pharmacol Sci 22: $110-117,2018$

64. Yu Y, Chen X and Cang S: Cancer-related long noncoding RNAs show aberrant expression profiles and competing endogenous RNA potential in esophageal adenocarcinoma. Oncol Lett 18 4798-4808, 2019.

65. Pan H, Guo C, Pan J, Guo D, Song S, Zhou Y and Xu D: Construction of a competitive endogenous RNA network and identification of Potential regulatory axis in gastric cancer. Front Oncol 9: 912, 2019

66. Peng J, Zhu Y, Dong X, Mao X, Lou Y, Mu Y, Xue D and Zhou H: Construction and analysis of IncRNA-associated ceRNA network identified potential prognostic biomarker in gastric cancer. Transl Cancer Res 8: 1116-1128, 2019.

67. Qi M, Yu B, Yu H and Li F: Integrated analysis of a ceRNA network reveals potential prognostic lncRNAs in gastric cancer. Cancer Med 9: 1798-1817, 2020.

This work is licensed under a Creative Commons Attribution-NonCommercial-NoDerivatives 4.0 International (CC BY-NC-ND 4.0) License. 